\title{
711.
}

\section{ON A DIAGRAM CONNECTED WITH THE TRANSFORMATION OF ELLIPTIC FUNCTIONS.}

[From the Report of the British Association for the Advancement of Science, (1881), p. 534.]

THE diagram relates to a known theorem, and is constructed as follows. Consider the infinite half-plane $y=+$; draw in it, centre the origin and radius unity, a semicircle; and draw the infinite half-lines $x=-\frac{1}{2}$, and $x=\frac{1}{2}$; then we have a region included between the lines, but exterior to the semicircle. The region in question may be regarded as a curvilinear triangle, with the angles $60^{\circ}, 60^{\circ}$, and $0^{\circ}$. The region may be moved parallel to itself in the direction of the axis of $x$, through the distance 1; say this is a "displacement"; $\sim r$ we may take the "image" of the region in regard to the semicircle. Performing any number of times, and in any order, these two operations of making the displacement and of taking the image, we obtain a new region, which is always a curvilinear triangle (bounded by circular arcs) and having the angles $60^{\circ}, 60^{\circ}, 0^{\circ}$; and the theorem is that the whole series of the new regions thus obtained completely covers, without interstices or overlapping, the infinite half-plane. The number of regions is infinite, and the size of the successive regions diminishes very rapidly. The diagram was a coloured one, exhibiting the regions obtained by a few of the successive operations.

The analytical theorem is that the whole series of transformations, $\omega$ into $\frac{\alpha \omega+\beta}{\gamma \omega+\delta}$, where $\alpha, \beta, \gamma, \delta$ are integers such that $\alpha \delta-\beta \gamma=1$, can be obtained by combination of the transformations $\omega$ into $\omega+1$ and $\omega$ into $-\frac{1}{\omega}$. 